\title{
MiRNAs and Their Interference with the Main Molecular Mechanisms Responsible for Drug Resistance in Pancreatic Cancer
}

\author{
Ingrid Garajová • Elisa Giovannetti • Sara Caponi • \\ Annette van Zweeden • Godefridus J. Peters
}

Published online: 15 January 2015

(C) Springer International Publishing AG 2015

\begin{abstract}
Pancreatic ductal adenocarcinoma (PDAC) is a genetically heterogeneous disease with a very limited response to most treatments. The main mechanisms responsible for drug resistance in PDAC include molecular aberrations in key regulatory genes or signaling pathways, the desmoplastic reaction which characterize pancreatic cancer microenvironment, and the presence of resistant cancer stem cells (CSCs). The recent discovery of microRNAs (miRNAs) provided new directions for research on all these mechanisms, demonstrating their
\end{abstract}

This article is part of the Topical Collection on miRNA and Cancer Prevention and Therapeutic Agents

I. Garajová • E. Giovannetti • A. van Zweeden • G. J. Peters $(\bowtie)$ Department of Medical Oncology, VU University Medical Center, Cancer Center Amsterdam, De Boelelaan 1117, 1081

HV Amsterdam, The Netherlands

e-mail: gj.peters@vumc.nl

I. Garajová

e-mail: ingegarajova@gmail.com

E. Giovannetti

e-mail: e.giovannetti@vumc.nl

A. van Zweeden

e-mail: a.vanzweeden@vumc.nl

I. Garajová

Department of Experimental, Diagnostic and Speciality Medicine,

University of Bologna, Sant'Orsola-Malpighi Hospital, Via

Massarenti 9, 40138 Bologna, Italy

E. Giovannetti

Start-Up Unit, University of Pisa, Lungarno Pacinotti 43, 56126 Pisa, Italy

\section{S. Caponi}

Unit Medical Oncology-2, Azienda Ospedaliero-Universitaria

Pisana, Via Roma, 67, Pisa, Italy

e-mail: saracaponi@hotmail.it important role in the regulation of many important biological processes, such as cell survival, proliferation, migration, invasion, and metastasis. Furthermore, there is increasing interest in defining a functional association between miRNAs and chemoresistance, with the double aim to rationally design miRNA-based strategies to improve clinical outcome and to identify novel biomarkers of treatment response. In this review, we summarize the current knowledge on the role of miRNAs and their interference with the main molecular mechanisms responsible for drug resistance in PDAC.

Keywords microRNAs · Pancreatic cancer · Drug resistance · Prognosis

\section{Introduction}

Pancreatic ductal adenocarcinoma (PDAC) is a highly aggressive malignancy and fourth leading cause of cancer-related death in developed countries [1]. The median survival after diagnosis is $2-8$ months, and approximately only $3-6 \%$ of all patients with PDAC survive 5 years after diagnosis. Surgical resection remains the cornerstone of management of PDAC, but this is only feasible for a limited number of patients. The average survival of resected patients is between 12 and 20 months, with a high probability of relapse. Since symptoms are not very clear in early stages, $80 \%$ of PDACs are diagnosed when already advanced, and no curative therapy is currently available [1]. Chemotherapy prolongs life by only a few months, and PDAC chemoresistance renders most drugs ineffective.

Cancer chemoresistance can occur by multiple mechanisms. It can arise from physiological barriers 
for drug absorption or penetration into target tissues or from biological mechanisms within individual tumor cells. The latter can reduce the effectiveness at their intended site of action, such as increased expression of enzymes involved in drug catabolism or antiapoptotic proteins [2•].

MicroRNAs (MiRNAs) are a class of short non-coding RNAs (ncRNAs) containing approximately 19-24 nt. MiRNAs regulate more than one third of all human genes, which demonstrates their remarkable influence on human biology [3]. It is known that more than $50 \%$ of miRNA genes are localized within genomic regions that are either frequently amplified or deleted in different tumor types, resulting in miRNA deregulation and aberrant expression $[4,5]$. The altered miRNAs may have different effects on the tumors [6]. Some of these miRNAs have been characterized as potent oncogenes (oncomiRs), while others have been identified as tumor suppressors (tsmiRs) based on the consequences of their expression on the phenotype of several experimental models [7]. OncomiRs, such as miR-21, are commonly upregulated in cancer [8], while tsmiRs, such as let-7, are downregulated [9], resulting in unique combinations of miRNAs (i.e., overexpressed oncomiRs and underexpressed tsmiRs) characterizing different tumors [10]. Thus, hypothetically, restoring the expression of some miRNAs in tumor cells could lead to differentiation and therefore present a promising therapeutic strategy. Most cancers have a specific miRNA signature, or "miRNome," that characterizes the malignant state and defines some of the clinico-pathological features of the tumors (e.g., grade, stage, aggressiveness, vascular invasion, and/or proliferation index) [11].

MiRNA expression levels can be detected in a variety of human specimens including both fresh and formalin-fixed paraffin-embedded tissues, fine needle aspirates, and in almost all human body fluids, including serum, plasma, saliva, urine, and amniotic fluid $[12,13]$. The impressive stability of miRNAs in tissues and biofluids is a key advantage over proteins and mRNAs.

The present review summarizes the current knowledge on the role of miRNAs and their interference with the main mechanisms responsible for drug resistance in pancreatic cancer.

\section{MiRNA and Their Involvement in PDAC Signaling Pathways}

At the molecular level, in PDAC, there is a high frequency of major driver mutations in key regulatory genes, including KRAS, TP53, CDKN2A, and SMAD4, and aberrant activation of multiple signaling pathways [14]. Importantly, several miRNAs functionally interact with these signaling pathways, as described in the following paragraphs (see also Fig. 1).
KRAS-EGFR Pathway

Over $90 \%$ of PDACs harbor an activating KRAS gene mutation. The vast majority of these mutations are at codon 12 and occur very early in pancreatic carcinogenesis [15]. Several recent studies have identified specific miRNAs that regulate the KRAS signaling pathway in pancreatic oncogenesis. Preclinical studies have shown that KRAS regulates miR-21 expression levels in precancerous pancreatic lesions, and the peak of miR-21 expression correlates with the degree of progression to more aggressive forms [16]. Another study identified KRAS as a direct target of miR-96 [17]. Indeed, overexpression of miR-96 decreased pancreatic cancer cell invasion, migration and slowed tumor growth, and was associated with KRAS downregulation [17]. MiR-126 and let-7d can also downregulate KRAS levels in PDAC. In particular, miR-126 can directly target KRAS; thus, miR-126 downregulation can allow overexpression of KRAS [18].

The K-RAS pathway is closely related and strictly interconnected to the EGFR pathway. Indeed, the human EGFR family is comprised of four members (EGFR/ErbB1, ErbB2 or Her2/neu, ErbB3, and ErbB4). Activated EGF receptors recruit multiple adaptor and effector proteins and then initiate signaling via the RAS-ERK, PI3K-AKT, or JAK-STAT pathways to regulate a multitude of cellular activities, such as cell division, differentiation, migration, and programmed cell death [19].

EGFR overexpression is present in more than $95 \%$ of PDAC patients [20]. Ali et al. demonstrated an association of miR-146a and overexpression of EGFR in PDAC cells. In addition, inhibition of EGFR by siRNA transfection in cells stably transfected with pre-miR-146a decreased cell invasion as well as decrease of EGFR expression [20]. Another miRNA involved in the EGFR pathway is miR200c which may directly inhibit the expression of the mitogen-inducible gene 6 (MIG6), a negative regulator of EGFR [21]. Moreover, it was demonstrated that the MIG6-mediated reduction of EGFR occurs concomitantly with activation of AKT signaling pathway, subsequently leading to an EGFR-independent phenotype that is refractory to EGFR tyrosine kinase inhibitors. Moreover, analyses of primary tumor xenografts of patientderived lung and pancreatic cancers carrying wild-type EGFR showed that the tumor MIG6(mRNA)/miR200 ratio is inversely correlated with response to erlotinib in vivo [21].

Up to $29 \%$ of PDACs present Her2 overexpression [22]. There is direct correlation between the expression levels of the Her2/neu and the shorter survival in PDAC patients, suggesting that the Her2/neu signaling pathway is a central regulator of PDAC oncogenesis [23, 24]. A recent study showed a relationship between miR-150 and Her2/neu [25]. The expression of miR-150 is downregulated in PDAC, suggesting that its restoration could serve as an effective approach for pancreatic cancer therapy. With a nanoparticle-based miR-150 

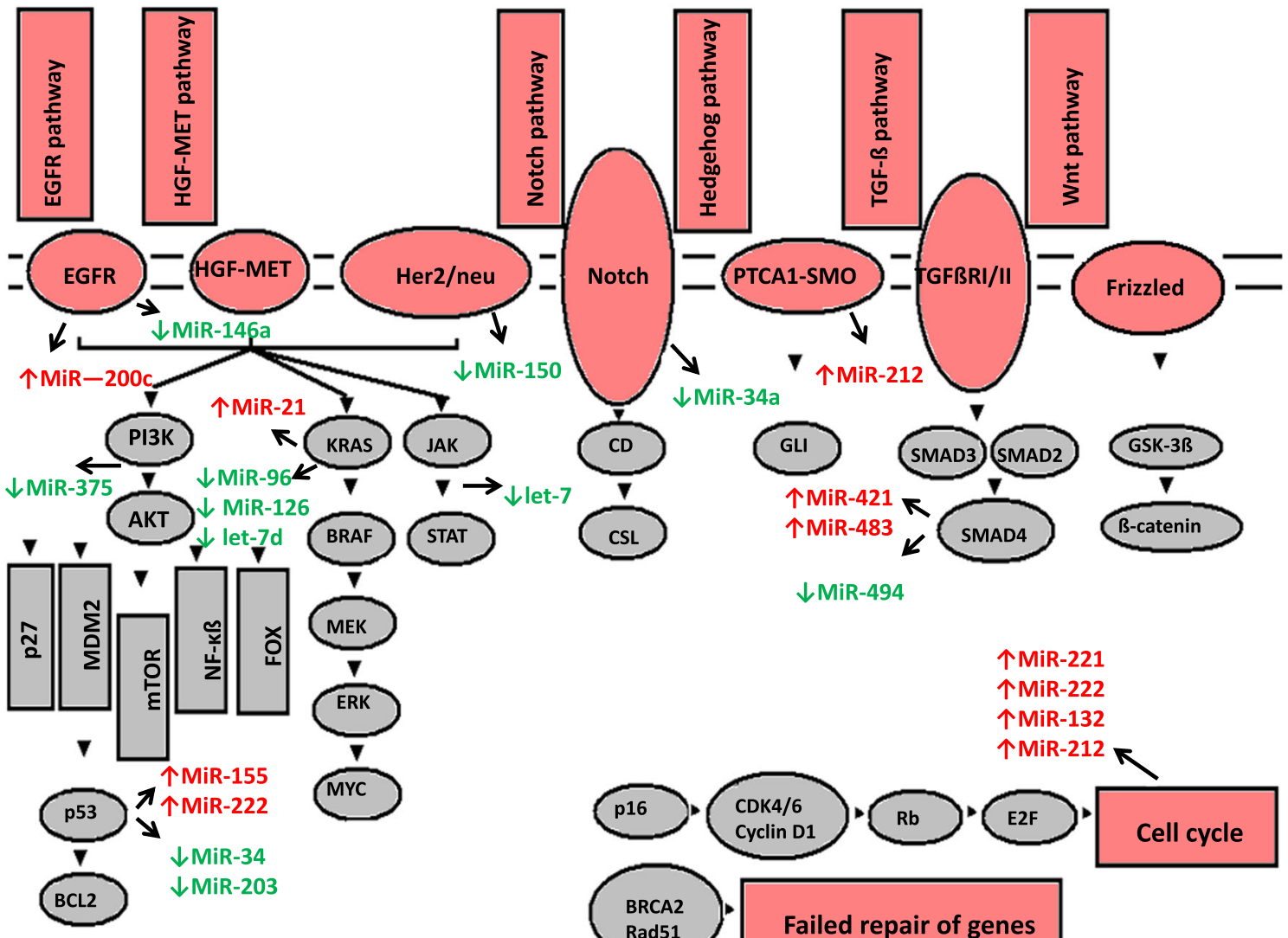

$\downarrow$ MiR-494

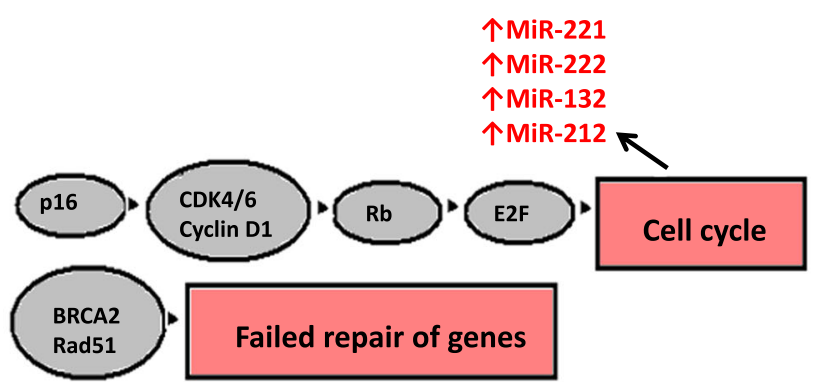

Fig. 1 MicroRNA and their involvement in oncogenic signaling pathways in PDAC. EGFR pathway, HGF-MET pathway, Notch pathway, Hedgehog pathway, TGF- $\beta$ pathway, and Wnt pathway with their downstream effector molecules. The effect of various miRs is indicated by its stimulation $(\uparrow)$ or inhibition $(\downarrow)$. EGFR pathway: Activation of the EGF receptor results in autophosphorylation of key tyrosine residues with subsequent activation of downstream signaling cascades including the RAS/extracellular signal-regulated kinase (ERK) pathway, the phosphatidylinositol 3-kinase (PI3) pathway, and the Janus kinase/signal transducer and activator of transcription (JAK/STAT) pathway. All of them result in cell survival promotion. HGF-MET pathway: The mesenchymal-epithelial transition factor gene (MET) is activated as a response to binding of its ligand, hepatocyte growth factor (HGF). Known effector molecules include the same ones as EGFR pathway. Notch pathway: A ligand on one cell induces a series of proteolytic cleavage events in a Notch receptor on a contacting cell. These cleavage events release the Notch intracellular domain (CD), which translocates to the nucleus to activate the transcription of Notch target genes together with CSL (CBF1/suppressor of hairless/LAG-1). The Notch signaling pathway is important for cell-cell communication, which involves gene regulation mechanisms that control multiple cell differentiation processes. Hedgehog pathway: Hedgehog is a secreted ligand that binds to its receptor, Patched (PTCA1). When PTCA1 is activated, it leads to inhibition of the Smoothened (Smo) receptor. Smo is then able to inhibit the phosphorylation and cleavage of Gli, which prevents the formation of repressive Gli (GliR) and promotes the formation of activated Gli (GliA). GliA then translocates into the nucleus and initiates transcription of target genes, which play a role in stem cell regulation. TGF- $\beta$ pathway: TGF receptors are activated after binding with their ligand, which leads to further phosphorylation of receptor-regulated SMADs (mainly SMAD2 and SMAD3). Phosphorylated SMAD2/3 forms heteromeric complexes with SMAD4, which accumulate in the nucleus and activate transcription of different genes, including those responsible for cell cycle arrest. Wnt pathway: In the absence of signal, action of the destruction complex (CKI $\alpha$, GSK $3 \beta$, APC, and Axin) creates a hyperphosphorylated $\beta$-catenin, which is a target for ubiquitination and degradation by the proteasome. Binding of Wnt ligand to a Frizzled/LRP-5/6 receptor complex leads to stabilization of hypophosphorylated $\beta$-catenin, which interacts with TCF/LEF proteins in the nucleus to activate transcription delivery system (miR-150-NF) tested in PDAC cells, an efficient intracellular delivery of miR-150 mimics was found, leading to a significant downregulation of its target gene (MUC4) expression. Inhibition of MUC4 correlated with a concomitant decrease in the expression of Her2/neu and repression of its downstream signaling in PDAC cells. These observations correlated with decreased growth and clonogenicity of PDAC cells and suppression of malignant behavioral properties [25].

\section{PI3K-AKT Signaling}

Activation of PI3K-AKT signaling is also correlated with malignant behavior in PDAC. miR-375 is downregulated in PDAC and negatively regulates the expression of 3phosphoinositide-dependent protein kinase 1 (PDK1) by directly targeting the $3^{\prime} \mathrm{UTR}$ of the PDK1 transcript. It may be concluded that miR-375 suppresses the malignant behavior of PDAC cells through the AKT signaling pathway [26]. 


\section{JAK-STAT Signaling}

Aberrant activation of the JAK-STAT pathway has been reported in a variety of disease states, including PDAC. Consistent downregulation of expression of miRNA let-7 is often observed in PDAC [27]. Re-expression of let-7 in poorly differentiated PDAC cell lines reduced phosphorylation/ activation of STAT3 and its downstream signaling events and reduced the growth and migration of PDAC cells. The mechanism is indirect: let-7 re-expression enhanced cytoplasmic expression of suppressor of cytokine signaling 3 (SOCS3), which blocks STAT3 activation by JAK2 [27].

\section{HGF-MET Pathway}

The mesenchymal-epithelial transition factor gene (MET) is activated as a response to binding of its ligand, hepatocyte growth factor (HGF). Known effector molecules of HGF-MET signaling cascade include Src, MEK-ERK1/2, PI3K-AKT, NK-kB, mTOR, and STAT [28, 29]. MET-mediated induction of these pathways will positively influence cell proliferation, migration, and survival [30]. An increased MET protein expression was found in PDAC compared to normal controls. Moreover, in PDAC, a correlation between MET protein overexpression and TNM stage was demonstrated [30].

Importantly, in pancreatic cancer stem cells (CSCs), MET overexpression conferred an equally tumorigenic phenotype in CD44+/CD24+/ESA+ cells [31 •]. An overexpression of MET has also been associated with epithelial-mesenchymal transition (EMT)-like changes in PDAC with acquired gemcitabine resistance [32]. miR-26a has been demonstrated to be lost in pancreatic CSCs, and its re-expression decreased expression of CSC markers (EpCAM and EZH2) in pancreatic cancer. In this respect, interestingly, pancreatic CSCs treated with metformin showed increased expression of six miRNAs including miR26a and decreased expression of different CSC markers [33].

\section{Notch Pathway}

Notch proteins are known type I transmembrane protein family members that function as receptors for membrane-bound ligands [34•]. The Notch signaling pathway is important for cell-cell communication, which involves gene regulation mechanisms that control multiple cell differentiation processes. Several studies have shown that the Notch signaling pathway is regulated by miRNAs during pancreatic oncogenesis. Re-expression of miR-34a inhibited cell growth and induced apoptosis, with concomitant downregulation of Notch-1 signaling pathway, one of the targets of miR-34a in PDAC cells [35]. Moreover, treatment of PDAC cells with genistein led to the upregulation of miR-34a, resulting in the downregulation of Notch-1, which was correlated with inhibition of cell growth and induction of apoptosis [35].
Hedgehog Pathway

The Hedgehog signaling pathway is deregulated in the majority of the PDACs [36]. Hedgehog is a secreted ligand that binds to its receptor, Patched1 (PTCA1). When PTCA1 is activated, it leads to inhibition of the Smoothened (Smo) receptor. Smo is then able to inhibit the phosphorylation and cleavage of Gli, which prevents the formation of repressive Gli (GliR) and promotes the formation of activated Gli (GliA). GliA then translocates into the nucleus and initiates transcription of target genes which play a role in stem cell regulation. MiR-212 is upregulated in PDAC tissues and cells which can downregulate PTCA1 in PDAC, implicating this signaling pathway in PDAC cell growth, migration, and invasion [37].

\section{TGF-ß Pathway}

TGF receptors are activated after binding with their ligand, which leads to further phosphorylation of receptor-regulated SMADs (mainly SMAD2 and SMAD3). Phosphorylated SMAD2/3 form heteromeric complexes with SMAD4, which accumulate in the nucleus and activate transcription of different genes, including those responsible for cell cycle arrest. This pathway is of key importance for PDAC [38].

The SMAD4 gene is inactivated in approximately $60 \%$ of PDACs [34•]. PDACs with loss of SMAD4 expression have higher rates of distant metastases and a poorer prognosis [39]. A recent study showed that loss of SMAD4 in PDAC cells was associated with increased levels of FOXM1, nuclear localization of $\beta$-catenin, and reduced levels of miR-494 [40]. Transgenic expression of miR-494 in PDAC cells produced the same effects as reducing expression of FOXM1 or blocking nuclear translocation of $\beta$-catenin, namely reduction of cell proliferation, migration, and invasion, and an increase of sensitivity to gemcitabine. Reduced expression of miR-494 correlated with increased PDAC metastasis and reduced survival times of patients. This study suggested that miR-494 might be a prognostic marker or a therapeutic target for patients with PDAC. The expression levels for two other miRs, miR-421 and miR-483$3 p$, were inversely correlated to SMAD4 expression in PDAC samples while ectopic expression of these miRNAs repressed SMAD4 protein levels in PDAC cell lines, suggesting that they are potent regulators of SMAD4 in PDAC [41, 42].

\section{Wnt Pathway}

In the absence of a signal, the action of the destruction complex (CKI $\alpha$, GSK3 $\beta$, APC, and Axin) creates a hyperphosphorylated $\beta$-catenin, which is a target for ubiquitination and degradation by the proteasome. Binding of Wnt ligand to a Frizzled/LRP-5/6 receptor complex leads to stabilization of hypophosphorylated $\beta$-catenin, which interacts with TCF/LEF proteins in the nucleus to activate transcription [43]. 
MiR-29a expression and the response to gemcitabine therapy in PDAC cells were related [43]. PDAC cells transfected with anti-miR-29a were more sensitive to gemcitabine with higher numbers of apoptotic cells and $\mathrm{S}$ phase accumulation compared to control cells. Moreover, the transfected cells showed lower activation of the $\mathrm{Wnt} / \beta$-catenin signaling pathway. The addition of Wnt3a (a Wnt/ $\beta$-catenin signaling activator) weakened the augmented growth inhibitory effect of anti-miR-29a transfection [43].

\section{p16/CDKN2A Inactivation}

Loss-of-function mutations in p16 (CDKN2A) occur in approximately $80 \%$ of sporadic PDAC [44]. Most of these inactivating mutations lead to loss of function of the protein $\mathrm{p} 16$, the product of the CDKN2A gene. The $\mathrm{p} 16$ protein binds cyclin-dependent kinases 4 and 6 (CDK4 and CDK6) and specifically inhibits their $\mathrm{pRb}$ phosphorylating activity, which is required for $\mathrm{G} 1 / \mathrm{S}$ transition [38]. Inherited mutations in the p16/CDKN2A gene cause the familial atypical multiple mole melanoma syndrome, with increased risk for developing PDAC and melanoma [45]. Several miRNAs that deregulate the cell cycle genes are essential during PDAC development and progression. For example, miR-222 targets p27 and p57, which are both pivotal cell cycle inhibitors [46]. Other studies have shown that downregulation of miR-132 and miR-212 causes G2/M cell cycle arrest and results in reduced cell proliferation [47].

\section{TP53 Mutations}

The TP53 gene is inactivated in 75 to $85 \%$ of PDACs [15]. Genetic inactivation of TP53 abrogates important cell functions, such as regulation of cellular proliferation and apoptosis in response to DNA damage. When cellular stress and DNA damage are detected, degradation of TP53 is inhibited by different mechanisms, leading to accumulation of its active form [48]. Preclinical studies have shown that TP53 directly regulates miR-34, which targets Notch, and therefore plays a role in the maintenance and survival of PDAC-initiating cells [48]. Moreover, TP53-induced nuclear protein 1 gene has been described to be downregulated by miR-155, accelerating pancreatic tumor development [49]. MiR-222 and miR-203 are also able to target p53 and affect its function as a crucial regulator of the cell cycle [50].

\section{BRCA2 and PALB2 Mutations}

The BRCA2 gene is inactivated in fewer than $10 \%$ of PDACs [51]. Importantly, germline mutations in BRCA2 are associated with an increased risk of PDAC [52]. Similarly, germline truncating mutations in the PALB2 gene, which encodes for a BRCA2-binding protein [53], have been identified in $\sim 3 \%$ of individuals with familial pancreatic cancer $[54,55]$. Of note, a recent study for the prediction of BRCA1/2 mutationassociated hereditary breast cancer identified a 35-miRNA classifier for the prediction of BRCA1/2 mutation status with a reported 95 and $92 \%$ accuracy in the training and the test set, respectively [56]. These miRNA signatures might be of interest also in PDAC in order to complement current patient selection criteria for gene testing by identifying individuals with high likelihood of being BRCA1/2 mutation carriers.

\section{MicroRNA-Based Mechanisms of Anticancer Drug Resistance}

Drug resistance can be divided into intrinsic and acquired resistance. Intrinsic resistance is caused by a pre-existing phenotype, whereas acquired resistance develops due to repeated use of the same drug. A most common form of resistance to a broad range of anticancer drugs is the overexpression of one or more energy-dependent transporters that detect and efflux anticancer drugs from cells, resulting in multidrug resistance (MDR) [5, 57]. However, drug resistance has many different aspects, including miRNAs. Several miRNAs alter cellular response to anticancer agents via modulation of drug efflux and drug targets, DNA repair, cell cycle, and/or apoptotic response, as reported in the following paragraphs (see also Table 1).

\section{Upregulation of Drug Efflux Transporters}

Drug efflux is an important form of resistance against many currently used antineoplastic agents. An increased expression of drug efflux pumps keeps the intracellular drug concentration below a cell-killing threshold [57]. These multidrug transporters belong to the ubiquitous superfamily of ATPbinding cassette $(\mathrm{ABC})$ proteins which modulate absorption, distribution, and excretion of many xenobiotics and natural

Table 1 Selected miRNA candidates which are correlated to anticancer drug resistance

\begin{tabular}{lll}
\hline $\begin{array}{l}\text { MiRNA-based } \\
\text { mechanisms of } \\
\text { anticancer drug } \\
\text { resistance }\end{array}$ & $\begin{array}{l}\text { Selected interfering } \\
\text { oncomiRs }\end{array}$ & $\begin{array}{l}\text { Selected } \\
\text { interfering } \\
\text { tsmiRs }\end{array}$ \\
\hline $\begin{array}{l}\text { Upregulation of drug } \\
\text { efflux transporters }\end{array}$ & $\begin{array}{c}\text { miR-181a-5p, miR- } \\
218-5 \mathrm{p}\end{array}$ & $\begin{array}{c}\text { miR-130a-3p and } \\
\text { miR-424-3p }\end{array}$ \\
$\begin{array}{c}\text { Alterations in drug } \\
\text { targets }\end{array}$ & miR-192, miR-215 & miR-211, let-7 \\
$\begin{array}{c}\text { Alterations in DNA } \\
\text { repair }\end{array}$ & miR-21 & \\
$\begin{array}{c}\text { Aberrant regulation } \\
\text { of the cell cycle }\end{array}$ & miR-221, miR-222 & miR-34a, miR-26a \\
$\begin{array}{c}\text { Evasion of apoptosis } \\
\text { miR-21, miR-148a, } \\
\text { miR-200, miR-17-5p }\end{array}$ & $\begin{array}{l}\text { let-7, miR-204, } \\
\text { miR-320 }\end{array}$ \\
\hline
\end{tabular}


and pharmacological compounds. The best known representatives of this family are P-glycoprotein (P-gp) (ABCB1 encoded by the MDRl gene) and the human multidrug resistance-associated proteins (MRP) family (ABCC1-12). MiRNAs have also been shown to be involved in chemotherapy resistance through the regulation of $\mathrm{ABC}$ membrane transporters [58].

The role of miRNAs in MDR in PDAC was investigated via monitoring the modulation of some specific miRNAs by the treatment of a wild-type cell line (MDCK cells) and in the corresponding cell line with P-gp overexpression, insensitive to several antineoplastic treatments (MDCK-MDR1 cells) [59]. In particular, MDCK cells were stimulated with a specific P-gp substrate and a selective P-gp inhibitor, verifying the down- or upregulation of miRNAs with respect to untreated cells. A different modulation of four miRNAs (miR-181a5p, miR-218-5p, miR-130a-3p, and miR-424-3p) was found. In particular, miRNA-424 was identified as downregulated in MDR1-MDCK cells stimulated with a specific P-gp substrate. MiR-424 mediates the induction of the hypoxia-inducing factors HIF- $1 \alpha$ with subsequent MDR1 upregulation. Moreover, miR-424 modulates also the expression of protein cullin 2 (CUL2), a scaffolding protein displaying a pivotal role in the assembly of the ubiquitin ligase system, thereby stabilizing HIF- $1 \alpha[59]$.

\section{Alterations in Drug Targets}

Chemoresistance can also be caused by either quantitative (i.e., modulation of expression levels) or qualitative (i.e., mutation) alterations of the drug targets [58]. Examples of quantitative alterations have been reported for several antimetabolites, which influence various steps in the metabolism of the building stones of nucleic acids, through inhibition of key enzymes, such as thymidylate synthase (TS) and ribonucleotide reductase (RR). MiR-192 and miR-215 target TS, which is the main drug target of the fluoropyrimidine-based therapy in colorectal cancer, which is also used in PDAC patients [60]. Two recent studies suggested a key role of miR-211 in the modulation of ribonucleotide reductase subunit 2 (RRM2), which is an important cellular target of gemcitabine. This miRNA had a higher expression in PDAC patients with long vs. short overall survival. MiR-211 was one of the key miRNAs when evaluating high-resolution miRNA profiles with the Toray's 3D Gene ${ }^{\mathrm{TM}}$ miRNA chip, detecting more than 1200 human miRNAs [61•]. Induction of miR-211 expression in PDAC cell lines increased the sensitivity to gemcitabine through reduced expression of its target RRM2 [62]. Similarly, it has been demonstrated that let-7 negatively regulates RRM2 and let-7 expression is inversely correlated with RRM2 expression in gemcitabine-resistant PDAC cells. Additionally, silencing RRM2 or overexpression of let-7 was shown to sensitize PDAC cells to gemcitabine [63].
Alterations in DNA Repair

MiRNAs can also alter cellular response to several anticancer drugs via interference with DNA repair. In particular, inhibition of RR by gemcitabine results in deoxyadenosine triphosphate (dATP) depletion, causing DNA replication errors. Moreover, gemcitabine is incorporated into DNA and arrests DNA replication. Both the mispaired bases and the gemcitabine-modified DNA bases can be the substrates for postreplicative DNA mismatch repair (MMR) machinery [64], which influences cancer cell sensitivity. In several papers, we described ribonucleotide (NTP) and deoxyribonucleotide (dNTP) levels and related disturbances with differences in effects between sensitive and resistant cell lines. We demonstrated that gemcitabine-induced concentration and combination dependent changes in NTP and dNTP pools $[65,66]$.

Defects in MMR proteins have been associated with reduced or absent benefit from 5-FU adjuvant chemotherapy [67]. MMR alterations reduce the incorporation into DNA of the 5-FU metabolites that cause G2/M arrest and induce apoptosis after 5-FU treatment. Colorectal cancer cells with miR-21 overexpression reduced 5-FU-induced G2/M damage arrest and apoptosis, suggesting that miR-21-dependent downregulation of core MMR component (hMSH2-hMSH6) might contribute to both primary and acquired resistance to 5FU [68]. Of note, miR-21 is included in the miRNA metasignature for recognizing PDAC [69•, 70•]. Furthermore, high miR-21, high miR-31, and low miR-375 tumoral expression have been validated as independent prognostic biomarkers for poor overall survival in PDAC [70•].

\section{Aberrant Regulation of the Cell Cycle}

Uncontrolled cellular proliferation is one of the hallmarks of cancer, and these alterations are commonly caused by genetic damages to regulator genes such p16 and cyclin D1 or the tumor suppressor TP53 [71].

Recent studies showed that the members of the miR-34 family are direct TP53 targets, and their upregulation induced apoptosis and cell cycle arrest [72]. Moreover, the promoter region of $\mathrm{miR}-34 \mathrm{a} / \mathrm{b} / \mathrm{c}$ contains $\mathrm{CpG}$ islands. $\mathrm{CpG}$ methylation reduces miR-34 family expression in multiple types of cancer, including PDAC [73]. Epigenetic restoration with chromatin modulators, demethylating agent 5-Aza-2'deoxycytidine and HDAC inhibitor Vorinostat [48], increased MiR-34a in PDAC. The restoration of miR-34a in human PDAC and CSCs strongly inhibited cell proliferation, cell cycle progression, self-renewal, epithelial-to-mesenchymal transition, and invasion, while inducing apoptosis. These results provided not only mechanistic insight but also promising therapeutic approaches, which might also improve the existing chemotherapies in PDAC. Denk et al. demonstrated that miR-26a is expressed in the cytoplasm of pancreatic 
ductal epithelial cells, whereas its expression was downregulated in PDAC tissues compared to that of adjacent benign pancreatic tissues. Patients with low miR-26a expression had a shorter survival than those with high miR-26a expression. The in vitro and in vivo assays showed that overexpression of miR-26a resulted in cell cycle arrest, inhibited cell proliferation, and decreased tumor growth, which was associated with cyclin E2 downregulation [74]. Another example of interaction between proteins regulating the cell cycle and miRNAs is represented by Cyclin-dependent kinase inhibitor 1B (CDKN1B or p27), which is a cell cycle inhibitor and tumor suppressor. This enzyme has been identified as a direct target of miR-221 and miR-222 [75]. The expression of miR-221 is upregulated in PDAC cell lines and tumor tissues compared to normal pancreatic duct epithelial cells and normal pancreas tissues and has been proposed as a candidate plasma biomarkers in PDAC [76]. However, transfection of miR-221 inhibitor suppressed the proliferative capacity of PDAC cells with concomitant upregulation of CDKN1B, as well as of PTEN and PUMA, which are other tumor suppressors among the predicted targets of miR-221 [77]. The same study showed that the expression of miR-221 was modulated by the treatment with isoflavone mixture (G2535), formulated 3,3'diindolylmethane (BR-DIM), or a synthetic curcumin analogue (CDF), leading to the inhibition of cell proliferation and migration and supporting further studies on these potential non-toxic agents in novel targeted therapeutic strategy that are capable of downregulation of miR-221.

\section{Evasion of Apoptosis}

Apoptotic evasion is considered to be one of the main causes of chemotherapeutic and radiotherapeutic resistance that characterizes the most aggressive tumor [78]. Cancer cells can resist apoptosis if they have an overexpression of antiapoptotic proteins involved in the two main apoptosis pathways, extrinsic and intrinsic. The extrinsic pathway is regulated mainly by "death receptors" of the TNF receptor family, while the intrinsic pathway is regulated by Bcl-2 proteins. Various anticancer drugs such as antimetabolites, DNA cross-linking and intercalating agents, alkylating agents, topoisomerase I/II inhibitors, and TKIs have been reported to induce intrinsic or extrinsic apoptotic response in tumor cells, resulting in caspase activation [79]. Although the extrinsic and the intrinsic apoptosis pathways are activated by different stimuli, both pathways can be regulated by specific miRNAs. For example, upregulation of Bcl-2, directly induced by miR21 , is associated with decreased apoptosis, chemoresistance to gemcitabine, and proliferation of MIA PaCa-2 cells [80]. Using Western blot and luciferase activity assay, $\mathrm{Bcl}-2$ was also identified as a target of miR-148a, and the expression of Bcl-2 lacking in 3'UTR could abrogate the proapoptotic function of miR-148a in PANC-1 and AsPC-1 cells [81]. Similarly, exogenous expression of miR-204 and miR-320 reduced the protein level of their targets, Bcl-2 and Mcl-1, respectively. Mcl-1 is an antiapoptotic member of Bcl-2 family, and induction of miR-320 activity led to apoptosis through Mcl-1 suppression, sensitizing cholangiocarcinoma cells to 5-FU [82]. However, miR-204 was also reported to be downregulated in gemcitabine-resistant PDAC [83], and Li et al. identified a role for the entire miR-200 family of miRNAs in gemcitabineresistant PDAC cells [84]. Conversely, miR-17-5p downregulates the proapoptotic member of the $\mathrm{Bcl}-2$ protein family Bim, and PDAC cells transfected with miR-17-5p inhibitor showed growth inhibition, spontaneous apoptosis, higher caspase- 3 activation, and increased chemosensitivity to gemcitabine [85]. Pathways delivering an antiapoptotic signal, such as PI3K/AKT, also play a pivotal role in the balance between proapoptotic and survival signals, which determine the fate of cancer cells. An increased miR-21 expression has been associated with the activation of PI3K/AKT/mTOR pathway, while combination of anti-miR-21 strategies with drugs targeting $\mathrm{PI} 3 \mathrm{~K} / \mathrm{AKT} / \mathrm{mTOR}$ pathway reduced $\mathrm{pAKT}$ levels and enhanced apoptosis when used in combination with gemcitabine [86]. Importantly, the antiapoptotic role of miR21 is possibly tumor specific since inhibition of miR-21 increased sensitivity and apoptosis induction by gemcitabine in PDAC and cholangiocarcinoma, but not in colon cancer cells [86]. This suggests that its oncogenic properties could be cell and tissue dependent and that its potential role in chemoresistance should be put in the context of the tumor type and the treatment [87].

Also, the extrinsic apoptotic pathway can be influenced by diverse miRNAs. The extrinsic pathway is regulated mainly by death receptors of the TNF receptor family. The tumor necrosis factor-related apoptosis inducing ligand (TRAIL)induced cell death has been implicated in tumor surveillance. It was demonstrated that nuclear TRAIL-R2 interacts with ribonucleoprotein complexes involved in the maturation of miRNAs of the let-7 family, key differentiation controlling [88]. Knockdown of TRAIL-R2 resulted in increased levels of mature let-7 and, consequently, in reduced abundance of the targets of let-7, high mobility group AT-hook protein 2 (HMGA2) and Lin28B, leading to decreased cell proliferation. Therefore, nuclear TRAIL-2 can contribute to malignancy [88].

\section{microRNA-Based Mechanism Associated with Microenvironment and Cancer Stem Cells Chemoresistance of PDAC}

PDAC is characterized by a dense fibrotic stromal matrix [89] composed of activated fibroblasts/stellate cells, inflammatory cells, and other cell types such as endothelial 
cells. PDAC is one of the most stroma-rich malignancies [90]. Such desmoplasia facilitates a mechanopathology known as growth-induced solid stress, resulting in collapsed or compressed intratumoral blood vessels or lymphatics, which lead to increased hypoxia and interstitial fluid pressure, both attenuating chemosensitivity [91]. MiR-210 is induced by hypoxia, and the levels of this miRNA are elevated in PDAC patients and may potentially serve as a useful biomarker for PDAC diagnosis [92]. Furthermore, miR-210 regulates the interaction between PDAC cells and stellate cells, promoting the progression and chemoresistance of tumor cells [93]. However, the same study showed that stellate cell-induced miR-210 upregulation was inhibited by inhibitors of ERK and PI3K/AKT pathways, suggesting novel therapeutic combinations to counteract the interaction between stellate cells and PDAC. This interaction is, at least in part, responsible for the innate resistance to chemotherapy in pancreatic tumors by creating barriers against circulating drugs. Hypoxia also induced the overexpression of miR-21 [94], while the treatment with the novel curcumin-derived analogue CDF downregulated the expression of miR-21 and miR210, as well as Nanog, Oct4, EZH2 mRNAs, and the production of VEGF and IL-6. CDF also led to decreased cell migration/invasion, angiogenesis, and formation of pancreatospheres under hypoxia, supporting further studies on its role to overcome microenvironment-mediated chemoresistance of PDAC [95].

The existence of CSCs has been widely accepted to be responsible for tumor aggressiveness in PDAC. By definition, stem cells have the capability of self-renewal to differentiate into each cell type and the potential to proliferate extensively [96]. Adult stem cells have been found in almost every tissue type and organ-maintaining tissue homeostasis and in regenerating tissues after injury or damage [97]. It is known that CSCs constitute only a small percentage $(0.05-1 \%)$ of tumor cells within a tumor mass containing heterogeneous population of tumor cells within the tumor microenvironment [98•].

Pancreatic CSC populations express the cell surface markers $\mathrm{CD} 44+, \mathrm{CD} 24+$, and epithelial-specific antigen (ESA)+ [99], while MET expression also seems to be an important marker [35]. The mechanism by which MET overexpression confers chemoresistance in pancreatic cancer likely involves the mesenchymal support network. Stroma is the predominant source of HGF, suggesting MET activation is, at least in part, a result of paracrine signaling [29].

It is also known that miRNAs regulate the CSC characteristics by affecting signaling pathways and CSC signature genes. A recent study detected deregulated expression of over 400 miRNAs, including let-7, miR-30, miR-125b, and miR335 in PDAC CD44+/CD133+/EpCAM+ (triple marker positive) CSCs [100•]. In the same study, as a proof of concept, knockdown of miR-125b resulted in the inhibition of tumor aggressiveness, consistent with the downregulation of CD44, EpCAM, EZH2, and snail. These results clearly suggest the importance of miRNAs in the regulation of CSCs characteristics, and their potential role as novel targets to improve therapeutic efficacy. Recently, it was also demonstrated that miR-1246 induced chemoresistance and was related to cancer stemness in pancreatic cancer cell lines via controlling CCNG2 [101].

Taking together, in pancreatic cancer, CSCs have the capacity for increased cell growth, cell migration/invasion, metastasis, and also treatment resistance.

\section{Conclusions and Future Perspectives}

PDAC is one of the most aggressive and lethal malignancies. The only treatment option for unresectable locally advanced or metastatic PDAC remains cytotoxic chemotherapy. Clinical trials evaluating the combinations with signal transductiontargeted therapies have been disappointing. Actually, the therapeutic options for metastatic PDAC include gemcitabine as monotherapy or in combinations (mainly with nab-paclitaxel) or FOLFIRINOX which represent the combination of 5-FU, leucovorin, irinotecan, and oxaliplatin [102]. The response to most treatments is limited or might be too toxic, especially FOLFIRINOX. Therefore, a continued search for new effective therapies, as well as biomarkers to assess the likelihood of response to these therapies, is ongoing.

MiRNAs are clearly involved in PDAC tumorigenesis; progression and recent evidence support their utility as promising biomarkers in cancer diagnosis, prognosis, and drug response/resistance. In clinical practice, the advantage of miRNAs is their stability in both formalin-fixed, paraffinembedded tumor tissues and biofluids which make them ideal as pre-treatment static biomarkers (miRNAs expression in tissue) or dynamic biomarkers during the treatment (in plasma/serum of the PDAC patients) with the hope to identify the characteristic modulation associated with good/poor response to chemotherapy. Therefore, in this review, we summarized the miRNAs' interference with the main mechanisms responsible for drug resistance in PDAC (changes in key regulatory genes/signaling pathways, pancreatic microenvironment, and highly resistant CSCs).

Nowadays, no conclusive evidence about clinical utility of miRNAs has been obtained. This could be explained mainly by heterogeneous material or diverse techniques used for examining the expression of miRNAs. More studies avoiding heterogeneity are required to evaluate the real importance of miRNAs as predictive biomarkers in PDAC. 


\section{Compliance with Ethics Guidelines}

Conflict of Interest Ingrid Garajová, Elisa Giovannetti, Sara Caponi, Annette van Zweeden, and Godefridus J. Peters declare that they have no conflict of interest.

Human and Animal Rights and Informed Consent This article does not contain any studies with human or animal subjects performed by any of the authors.

\section{References}

Papers of particular interest, published recently, have been highlighted as:

-Of importance

1. Mohammed S et al. Pancreatic cancer: advances in treatment. World J Gastroenterol. 2014;20(28):9354-60.

2. Giovannetti E et al. Molecular mechanisms underlying the role of microRNAs (miRNAs) in anticancer drug resistance and implications for clinical practice. Crit Rev Oncol Hematol. 2012;81(2): 103-22. A very nice review about the role of microRNAs in anticancer drug resistance.

3. Bhardwaj A et al. MicroRNA-based cancer therapeutics: big hope from small RNAs. Mol Cell Pharmacol. 2010;2(5):213-9.

4. Calin GA et al. MicroRNA signatures in human cancers. Nat Rev Cancer. 2006;6(11):857-66.

5. Bhatti I et al. Small RNA: a large contributor to carcinogenesis? J Gastrointest Surg. 2009;13(7):1379-88.

6. Sethi $\mathrm{S}$ et al. MicroRNAs in personalized cancer therapy. Clin Genet. 2014;86(1):68-73.

7. Khan $\mathrm{S}$ et al. Targeting microRNAs in pancreatic cancer: microplayers in the big game. Cancer Res. 2013;73(22):6541-7.

8. Ali S et al. Gemcitabine sensitivity can be induced in pancreatic cancer cells through modulation of miR-200 and miR-21 expression by curcumin or its analogue CDF. Cancer Res. 2010;70(9): 3606-17.

9. Kong D et al. Loss of let-7 up-regulates EZH2 in prostate cancer consistent with the acquisition of cancer stem cell signatures that are attenuated by BR-DIM. PLoS One. 2012;7(3):e33729.

10. Gounaris-Shannon $\mathrm{S}$ et al. The role of miRNA in haematological malignancy. Bone Marrow Res. 2013;2013:269107.

11. Ji Q et al. MicroRNA miR-34 inhibits human pancreatic cancer tumor-initiating cells. PLoS One. 2009;4(8):e6816.

12. Zöller $\mathrm{M}$ et al. Pancreatic cancer diagnosis by free and exosomal miRNA. World J Gastrointest Pathophysiol. 2013;4(4):74-90.

13. Humeau $\mathrm{M}$ et al. miRNA in clinical practice: pancreatic cancer. Clin Biochem. 2013;46(10-11):933-6.

14. Ouyang $\mathrm{H}$ et al. microRNA-10b enhances pancreatic cancer cell invasion by suppressing TIP30 expression and promoting EGF and TGF- $\beta$ actions. Oncogene. 2013. doi:10.1038/onc.2013.405.

15. Jones $\mathrm{S}$ et al. Core signaling pathways in human pancreatic cancers revealed by global genomic analyses. Science. 2008;321(5897):1801-6.

16. du Rieu MC et al. MicroRNA-21 is induced early in pancreatic ductal adenocarcinoma precursor lesions. Clin Chem. 2010;56(4): $603-12$

17. Yu S et al. miRNA-96 suppresses KRAS and functions as a tumor suppressor gene in pancreatic cancer. Cancer Res. 2010;70(14): $6015-25$.
18. Jiao LR et al. MicroRNAs targeting oncogenes are downregulated in pancreatic malignant transformation from benign tumors. PLoS One. 2012;7(2):e32068.

19. Gomez GG et al. Therapeutic resistance in cancer: microRNA regulation of EGFR signaling networks. Cancer Biol Med. 2013;10(4):192-205.

20. Ali $\mathrm{S}$ et al. Deregulation of miR-146a expression in a mouse model of pancreatic cancer affecting EGFR signaling. Cancer Lett. 2014;351(1):134-42.

21. Izumchenko E et al. The TGF $\beta$-miR200-MIG6 pathway orchestrates the EMT-associated kinase switch that induces resistance to EGFR inhibitors. Cancer Res. 2014;74(14):3995-4005.

22. Yan $\mathrm{M}$ et al. HER 2 aberrations in cancer: implications for therapy. Cancer Treat Rev. 2014;40(6):770-80.

23. Komoto $\mathrm{M}$ et al. HER2 overexpression correlates with survival after curative resection of pancreatic cancer. Cancer Sci. 2009;100(7):1243-7.

24. Chou A et al. Clinical and molecular characterization of HER2 amplified-pancreatic cancer. Genome Med. 2013;5(8):78.

25. Arora $\mathrm{S}$ et al. Synthesis, characterization, and evaluation of poly (D, L-lactide-co-glycolide)-based nanoformulation of miRNA150: potential implications for pancreatic cancer therapy. Int $\mathrm{J}$ Nanomedicine. 2014;9:2933-42.

26. Zhou J et al. MicroRNA-375 targets PDK1 in pancreatic carcinoma and suppresses cell growth through the Akt signaling pathway. Int J Mol Med. 2014;33(4):950-6.

27. Patel K et al. MicroRNA let-7 downregulates STAT3 phosphorylation in pancreatic cancer cells by increasing SOCS3 expression. Cancer Lett. 2014;347(1):54-64.

28. Trusolino L et al. MET signalling: principles and functions in development, organ regeneration and cancer. Nat Rev Mol Cell Biol. 2010;11(12):834- 48.

29. Delitto $\mathrm{D}$ et al. c-Met signaling in the development of tumorigenesis and chemoresistance: potential applications in pancreatic cancer. World J Gastroenterol. 2014;20(26):8458-70.

30. Zhu GH et al. Expression and prognostic significance of CD151, c-Met, and integrin alpha3/alpha6 in pancreatic ductal adenocarcinoma. Dig Dis Sci. 2011;56(4):1090-8.

31. Li C et al. c-Met is a marker of pancreatic cancer stem cells and therapeutic target. Gastroenterology. 2011;141(6):2218-2227.e5. The authors explain in their article the role of c-met in PDAC CSCs.

32. Shah AN et al. Development and characterization of gemcitabineresistant pancreatic tumor cells. Ann Surg Oncol. 2007;14(12): 3629-37.

33. Bao $\mathrm{B}$ et al. Metformin inhibits cell proliferation, migration and invasion by attenuating CSC function mediated by deregulating miRNAs in pancreatic cancer cells. Cancer Prev Res (Phila). 2012;5(3):355-64.

34. Drakaki A et al. MicroRNA-gene signaling pathways in pancreatic cancer. Biomed J. 2013;36(5):200-8. An overview about how microRNAs are interfering with the signaling pathways in pancreatic cancer.

35. Xia $\mathrm{J}$ et al. Genistein inhibits cell growth and induces apoptosis through up-regulation of miR-34a in pancreatic cancer cells. Curr Drug Targets. 2012;13(14):1750-6.

36. Dosch JS et al. Pancreatic cancer and hedgehog pathway signaling: new insights. Pancreatology. 2010;10(2-3):151-7.

37. Ma $\mathrm{C}$ et al. miR-212 promotes pancreatic cancer cell growth and invasion by targeting the hedgehog signaling pathway receptor patched-1. J Exp Clin Cancer Res. 2014;33:54.

38. Partensky C. Toward a better understanding of pancreatic ductal adenocarcinoma: glimmers of hope? Pancreas. 2013;42(5):729-39.

39. Blackford A et al. SMAD4 gene mutations are associated with poor prognosis in pancreatic cancer. Clin Cancer Res. 2009;15(14):4674-9. 
40. Li L et al. Down-regulation of microRNA-494 via loss of SMAD4 increases FOXM1 and $\beta$-catenin signaling in pancreatic ductal adenocarcinoma cells. Gastroenterology. 2014;147(2):485-97.

41. Hao J et al. MicroRNA 421 suppresses DPC4/Smad4 in pancreatic cancer. Biochem Biophys Res Commun. 2011;406(4):552-7.

42. Hao $J$ et al. MicroRNA 483-3p suppresses the expression of DPC4/Smad4 in pancreatic cancer. FEBS Lett. 2011;585(1): 207-13.

43. Nagano $\mathrm{H}$ et al. MicroRNA-29a induces resistance to gemcitabine through the $\mathrm{Wnt} / \beta$-catenin signaling pathway in pancreatic cancer cells. Int J Oncol. 2013;43(4):1066-72.

44. Heilmann AM et al. CDK4/6 and IGF1 receptor inhibitors synergize to suppress the growth of p16INK4A-deficient pancreatic cancers. Cancer Res. 2014;74(14):3947-58.

45. Rustgi AK et al. Familial pancreatic cancer: genetic advances. Genes Dev. 2014;28(1):1-7.

46. Medina $\mathrm{R}$ et al. MicroRNAs 221 and 222 bypass quiescence and compromise cell survival. Cancer Res. 2008;68(8):2773-80.

47. Park JK et al. miR-132 and miR-212 are increased in pancreatic cancer and target the retinoblastoma tumor suppressor. Biochem Biophys Res Commun. 2011;406(4):518-23.

48. Nalls $\mathrm{D}$ et al. Targeting epigenetic regulation of miR-34a for treatment of pancreatic cancer by inhibition of pancreatic cancer stem cells. PLoS One. 2011;6(8):e24099.

49. Gironella $\mathrm{M}$ et al. Tumor protein 53- induced nuclear protein 1 expression is repressed by miR-155, and its restoration inhibits pancreatic tumor development. Proc Natl Acad Sci U S A. 2007;104(41):16170-5.

50. Greither T et al. Elevated expression of microRNAs 155, 203, 210 and 222 in pancreatic tumors is associated with poorer survival. Int J Cancer. 2010;126(1):73-80.

51. Goggins $\mathrm{M}$ et al. Germline BRCA2 gene mutations in patients with apparently sporadic pancreatic carcinomas. Cancer Res. 1996;56(23):5360-4.

52. Lal $\mathrm{G}$ et al. Inherited predisposition to pancreatic adenocarcinoma: role of family history and germ-line p16, BRCA1, andBRCA2 mutations. Cancer Res. 2000;60(2):409-16.

53. Xia B et al. Control of BRCA2 cellular and clinical functions by a nuclear partner, PALB2. Mol Cell. 2006;22(6):719-29.

54. Slater EP et al. PALB2 mutations in European familial pancreatic cancer families. Clin Genet. 2010;78(5):490-4.

55. Jones $\mathrm{S}$ et al. Exomic sequencing identifies PALB2 as a pancreatic cancer susceptibility gene. Science. 2009;324(5924):217.

56. Tanic $\mathrm{M}$ et al. MicroRNA expression signatures for the prediction of BRCA1/2 mutation-associated hereditary breast cancer in paraffin-embedded formalin-fixed breast tumors. Int $\mathrm{J}$ Cancer. 2014. doi:10.1002/ijc.29021.

57. Gottesman MM et al. Multidrug resistance in cancer: role of ATPdependent transporters. Nat Rev Cancer. 2002;2(1):48-58.

58. Garofalo $\mathrm{M}$ et al. MicroRNAs as therapeutic targets in chemoresistance. Drug Resist Updat. 2013;16(3-5):47-59.

59. Gisel A et al. miRNAs for the detection of multidrug resistance: overview and perspectives. Molecules. 2014;19(5):5611-23.

60. Boni Vet al. miR-192/miR-215 influence 5-fluorouracil resistance through cell cycle-mediated mechanisms complementary to its post-transcriptional thymidylate synthase regulation. Mol Cancer Ther. 2010;9(8):2265-75.

61. Giovannetti E et al. High-throughput microRNA (miRNAs) arrays unravel the prognostic role of MiR-211 in pancreatic cancer. PLoS One. 2012;7(11):e49145. The study about the prognostic role of miR-211 in pancreatic cancer.

62. Maftouh M et al. miR-211 modulates gemcitabine activity through downregulation of ribonucleotide reductase and inhibits the invasive behavior of pancreatic cancer cells. Nucleosides Nucleotides Nucleic Acids. 2014;33(4-6):384-93.
63. Bhutia YD et al. Differential processing of let-7a precursors influences RRM2 expression and chemosensitivity in pancreatic cancer: role of LIN-28 and SET oncoprotein. PLoS One. 2013;8(1):e53436.

64. Matthaios D et al. Molecular pathogenesis of pancreatic cancer and clinical perspectives. Oncology. 2011;81(3-4):259-72.

65. Van Moorsel CJA et al. Effect of gemcitabine and cis-platinum combinations on ribonucleotide and deoxyribonucleotide pools in ovarian cancer cell lines. Int J Oncol. 2003;22(1):201-7.

66. Peters GJ et al. Effects of gemcitabine on cis-platinum-DNA adduct formation and repair in a panel of gemcitabine and cisplatin-sensitive or -resistant human ovarian cancer cell lines. Int J Oncol. 2006;28(1):237-44.

67. Ribic $\mathrm{CM}$ et al. Tumor microsatellite-instability status as a predictor of benefit from fluorouracil-based adjuvant chemotherapy for colon cancer. N Engl J Med. 2003;349(3):247-57.

68. Valeri $\mathrm{N}$ et al. MicroRNA-21 induces resistance to 5- fluorouracil by down-regulating human DNA MutS homolog 2 (hMSH2). Proc Natl Acad Sci U S A. 2010;107(49):21098-103.

69. Frampton AE et al. A microRNA meta-signature for pancreatic ductal adenocarcinoma. Expert Rev Mol Diagn. 2014;14(3):26771. miRNA meta-signature for recognizing PDAC.

70. Ma MZ et al. Candidate microRNA biomarkers of pancreatic ductal adenocarcinoma: meta-analysis, experimental validation and clinical significance. J Exp Clin Cancer Res. 2013;32:71. A comprehensive meta-review of published studies in PDAC that compared the miRNA expression profiles of PDAC tissues and paired neighboring noncancerous pancreatic tissues was performed to determine candidate miRNA biomarkers for PDAC.

71. Sherr CJ et al. The Pezcoller lecture: cancer cell cycles revisited. Cancer Res. 2000;60(14):3689-95.

72. Bommer GT et al. p53- mediated activation of miRNA34 candidate tumor-suppressor genes. Curr Biol. 2007;17(15):1298-307.

73. Vogt $\mathrm{M}$ et al. Frequent concomitant inactivation of miR-34a and $\mathrm{miR}-34 \mathrm{~b} / \mathrm{c}$ by $\mathrm{CpG}$ methylation in colorectal, pancreatic, mammary, ovarian, urothelial, and renal cell carcinomas and soft tissue sarcomas. Virchows Arch. 2011;458(3):313-22.

74. Deng $\mathrm{J}$ et al. The loss of miR-26a-mediated post- transcriptional regulation of cyclin E2 in pancreatic cancer cell proliferation and decreased patient survival. PLoS One. 2013;8(10):e76450.

75. le Sage $\mathrm{C}$ et al. Regulation of the p27(Kip1) tumor suppressor by miR-221 and miR-222 promotes cancer cell proliferation. EMBO J. 2007;26(15):3699-708.

76. Kawaguchi $\mathrm{T}$ et al. Clinical impact of circulating miR-221 in plasma of patients with pancreatic cancer. Br J Cancer. 2013;108(2):361-9.

77. Sarkar $\mathrm{S}$ et al. Down-regulation of miR-221 inhibits proliferation of pancreatic cancer cells through up-regulation of PTEN, p27(kip1), p57(kip2), and PUMA. Am J Cancer Res. 2013;3(5):465-77.

78. Hanahan D et al. The hallmarks of cancer. Cell. 2000;100(1):57-70.

79. Kaufmann SH et al. Induction of apoptosis by cancer chemotherapy. Exp Cell Res. 2000;256(1):42-9.

80. Dong $\mathrm{J}$ et al. Bcl-2 upregulation induced by miR-21 via a direct interaction is associated with apoptosis and chemoresistance in MIA PaCa-2 pancreatic cancer cells. Arch Med Res. 2011;42(1):8-14.

81. Zhang $\mathrm{R}$ et al. MiR-148a regulates the growth and apoptosis in pancreatic cancer by targeting CCKBR and Bcl-2. Tumour Biol. 2014;35(1):837-44.

82. Chen $\mathrm{L}$ et al. The role of microRNA expression pattern in human intrahepatic cholangiocarcinoma. J Hepatol. 2009;50(2):358-69.

83. Ohuchida $\mathrm{K}$ et al. MicroRNA expression as a predictive marker for gemcitabine response after surgical resection of pancreatic cancer. Ann Surg Oncol. 2011;18(8):2381-7.

84. Li Y et al. Up-regulation of miR-200 and let-7 by natural agents leads to the reversal of epithelial-to-mesenchymal transition in gemcitabine-resistant pancreatic cancer cells. Cancer Res. 2009;69(16):6704-12. 
85. Yan HJ, et al. miR-17-5p inhibitor enhances chemosensitivity to gemcitabine via upregulating Bim expression in pancreatic cancer cells. Dig Dis Sci. 2012;57:3160-7.

86. Giovannetti E et al. MicroRNA-21 in pancreatic cancer: correlation with clinical outcome and pharmacologic aspects underlying its role in the modulation of gemcitabine activity. Cancer Res. 2010;70(11):4528-38.

87. $\mathrm{Li} \mathrm{J}$ et al. MiR-21 indicates poor prognosis in tongue squamous cell carcinomas as an apoptosis inhibitor. Clin Cancer Res. 2009;15(12):3998-4008.

88. Haselmann V et al. Nuclear death receptor TRAIL-R2 inhibits maturation of let-7 and promotes proliferation of pancreatic and other tumor cells. Gastroenterology. 2014;146(1):278-90.

89. Neesse A et al. Stromal biology and therapy in pancreatic cancer. Gut. 2011;60(6):861-8.

90. Feig $\mathrm{C}$ et al. The pancreas cancer microenvironment. Clin Cancer Res. 2012;18(16):4266-76.

91. Stylianopoulos $\mathrm{T}$ et al. Causes, consequences, and remedies for growth-induced solid stress in murine and human tumors. Proc Natl Acad Sci U S A. 2012;109(38):15101-8.

92. Ho AS et al. Circulating miR-210 as a novel hypoxia marker in pancreatic cancer. Transl Oncol. 2010;3(2):109-13.

93. Takikawa $\mathrm{T}$ et al. miR- 210 regulates the interaction between pancreatic cancer cells and stellate cells. Biochem Biophys Res Commun. 2013;437(3):433-9.

94. Mace TA et al. Hypoxia induces the overexpression of microRNA 21 in pancreatic cancer cells. J Surg Res. 2013;184(2):855-60.
95. Bao B et al. Hypoxia-induced aggressiveness of pancreatic cancer cells is due to increased expression of VEGF, IL-6 and miR-21, which can be attenuated by CDF treatment. PLoS One. 2012;7(12):e50165.

96. Tanase CP et al. Cancer stem cells: Involvement in pancreatic cancer pathogenesis and perspectives on cancer therapeutics. World J Gastroenterol. 2014;20(31):10790-801.

97. Raza $U$ et al. MicroRNAs: master regulators of drug resistance, stemness, and metastasis. J Mol Med (Berl). 2014;92(4):321-36.

98. Bao B, et al. Overview of cancer stem cells (CSCs) and mechanisms of their regulation: implications for cancer therapy. Curr Protoc Pharmacol. 2013; Chapter 14:Unit 14.25. A very nice overview about CSCs and mechanisms of their regulation.

99. Lee CJ et al. Pancreatic cancer stem cells. J Clin Oncol. 2008;26(17):2806-12.

100. Bao B et al. Differentially expressed miRNAs in cancer stem- like cells: markers for tumor cell aggressiveness of pancreatic cancer. Stem Cells Dev. 2014;23(16):1947-58. Results of this study clearly suggest the importance of miRNAs in the regulation of CSLC characteristics, and may serve as novel targets for therapy.

101. Hasegawa S et al. MicroRNA-1246 expression associated with CCNG2-mediated chemoresistance and stemness in pancreatic cancer. Br J Cancer. 2014;111(8):1572-80.

102. Garajová I et al. Molecular mechanisms underlying the role of MicroRNAs in the chemoresistance of pancreatic cancer. Biomed Res Int. 2014;2014:678401. 